\title{
Il trauma cranico da abuso (sindrome del bambino scosso): un vademecum per il pediatra
}

\author{
Melissa Rosa-Rizzotto, Martina Bua, Paola Facchin \\ Centro Regionale per la Diagnostica del Bambino Maltrattato, Azienda Ospedaliera Universitaria di Padova
}

Il trauma cranico da abuso (AHT, Abusive Head Trauma), comprensivo della sottoforma più nota, la sindrome del bambino scosso (SBS), è da tempo considerato tra le forme più severe di maltrattamento fisico nei primi mesi e anni di vita ed è annoverato tra le cause più frequenti di morte o di gravi disabilità nella primissima infanzia.

Una diagnosi precoce è essenziale per limitare i danni e prevenire eventi infausti, tuttavia è molto impegnativa: gli studi infatti riportano in media tre accessi ai servizi prima della diagnosi. Molte volte il bambino giunge all'attenzione dei servizi di emergenza e urgenza dopo ripetuti episodi di scuotimento e spesso si presenta in fase di esordio con sintomi subdoli che vengono scambiati per patologie banali o sintomi comuni. L'azione dello scuotere è in genere legata a una condizione di ridotta tolleranza allo stress per ragioni individuali, familiari, relazionali o contestuali che sommate insieme facilitano il passaggio all'atto e, il più delle volte, non è frutto di una condotta legata a un'ideazione a ledere quanto piuttosto a una reazione di tipo impulsivo.

Il pediatra di famiglia rappresenta il primo "nodo della rete" che può intercettare precocemente questa diagnosi, valutando i bambini e le famiglie fin dalla nascita, e rappresenta il primo professionista che può giocare un ruolo sia nella prevenzione primaria che nella riduzione della morbilità e mortalità dell'AHT attraverso la formazione e l'orientamento anticipatorio dei genitori rispetto ai rischi legati alla condizione dello scuotere, allo sviluppo normale del bambino e al comportamento del lattante nei primi mesi di vita - per esempio un'informazione chiara sul periodo del pianto come suggerito dal programma "PURPLE crying" (vedi infra) - oltre che a incoraggiare l'utilizzo di strategie di riduzione dello stress personale e familiare ed esempi di strategie comportamentali nei momenti di massima tensione, soprattutto in relazione al pianto.

Il presente lavoro, oltre a descrivere gli ele- menti clinici caratterizzanti la diagnosi, si propone di indicare quali particolari condizioni di salute dell'adulto o del bambino rendono il nucleo familiare complessivamente più vulnerabile e maggiormente soggetto a rischio di comportamenti impulsivi.

\section{Il problema definitorio}

Già più di quarant'anni or sono Norman Guthkelch propose che traumi con colpo di frusta nel bambino potessero causare un sanguinamento per un meccanismo di lacerazione delle vene a ponte dello spazio subdurale.

Si deve tuttavia a un radiologo pediatrico, John Caffey, l'aver individuato alcuni anni dopo il complesso quadro clinico che venne per la prima volta descritto, coniando una vera e propria sindrome specifica ("the whiplash shaken infant syndrome"), nell'articolo The whiplash shaken infant syndrome: manual shaking by the extremities with whiplash-induced intracranial and intraocular bleedings, linked with residual permanent brain damage and mental retardation, pubblicato nel 1974 sulla rivista Pediatrics. Tale prima denominazione, che comprendeva il termine whiplash la cui traduzione è "colpo di frusta", descriveva una serie di sintomi clinici ed emorragie in diversi distretti che si riscontravano con una minima e talvolta con nessuna evidenza esterna di trauma (sia al capo che nei restanti distretti corporei) e che derivavano dallo shaking, ovvero dall'atto dello scuotere manualmente il bambino dalle estremità.

L'evoluzione delle conoscenze e della tecnologia nella diagnostica per immagini ha permesso nel corso degli anni di migliorare l'abilità nella diagnosi di questa sindrome e ha acceso il dibattito rispetto alla necessità di raggruppare all'interno di un unico gruppo nosologico altre sindromi da maltrattamento che esitassero in una lesione al capo, non unicamente o esclusivamente per meccanismo di scuotimento. Così, già a partire dalla fine degli anni Novanta, in letteratura, sempre più clinici e ricercatori hanno scelto di utilizzare le espressioni "trauma cranico abusivo" o "trauma cranico inflitto" e nel 2009 l'American Academy of Pediatrics Committee on Child Abuse and Neglect ha raccomandato l'utilizzo di tale denominazione piuttosto che altre denominazioni impiegate sino a quel momento - Nonaccidental Head Injury (NAHI), Inflicted Traumatic Brain Injury (iTBI), Nonaccidental Head Trauma (NAHT), Shaken Impact Syndrome (SIS) - superando l'empasse di dover intendere un unico meccanismo di danno, come la sindrome del bambino scosso che esprimerebbe unicamente l'atto dello scuotere, e comprendendo anche il trauma chiuso (blunt trauma) o le asfissie, i soffocamenti e gli strangolamenti. Gli autori del presente lavoro tuttavia hanno ritenuto di mantenere la denominazione SBS sia perché tale dicitura è indicata nella nosologia ufficiale secondo l'International Classification of Diseases 9-Clinical Modification (ICD 9-CM) sia per la maggiore diffusione di tale termine in Italia.

\section{L'eziologia del danno: brevi considerazioni biomeccaniche e cinetiche}

Sul versante eziologico, questa forma di maltrattamento si perpetra attraverso un'azione di scuotimento violento del bambino, che può essere associato o meno ad altri meccanismi di lesione, come un trauma chiuso del capo con impatto su superficie o contro oggetto o ancora la compressione associata delle vie aeree.

Il tipo di movimento descritto, come già detto, genera gravi lesioni intracraniche per la rapida e ripetitiva flessione, estensione e rotazione del capo e del collo, con numerose e ripetute accelerazioni e decelerazioni repentine attorno a una struttura rigida e in genere mantenuta fissa: il tronco. Da questa descrizione si intende facilmente per quale motivo Caffey avesse scelto inizialmente una denominazione che faceva riferimento al colpo di frusta. Nel neonato il capo e il collo sono strutture mobili e flessibili, che egli/ella è scarsamente in grado di controllare per com- 
petenza e per volume e dimensione oltre che per disproporzione rispetto al resto del corpo, risultando particolarmente vulnerabili al momento dello scuotimento. Anche se evidenziato più di rado, gli autori ritengono doveroso sottolineare che tutto lo scheletro assile è sottoposto alle medesime sollecitazioni nell'atto dello scuotere e che tali sollecitazioni sono spesso causa di lesioni associate non sempre clinicamente manifeste, ma da ricercarsi con specifica diagnostica, nel caso si sospetti la sindrome. Il riferimento riguarda in particolare le oscillazioni flesso-estensorie del cingolo inferiore che causano sanguinamenti a livello spinale subdurale, oppure le oscillazioni disordinate e violente degli arti superiori o inferiori che possono causare le fratture metafisarie classiche da stiramento passivo.

Solitamente colui che scuote afferra il bimbo per il torace avvolgendo con le mani la gabbia toracica, per le braccia afferrando in genere il trat to omerale, per le spalle, per le gambe facendo oscillare tronco e capo oppure per il capo, e lo scuote vigorosamente e violentemente con flesso-estensioni rapide e ripetitive, a una frequenza di 2-4 volte al secondo per un periodo che varia da pochi secondi fino a circa 20 secondi.

Al termine di tale sequenza di movimenti si può associare un impatto: il bambino può essere impattato contro un oggetto (es. il fasciatoio) oppure può essere gettato con forza contro una superficie (es. il pavimento), associando le conseguenze dello scuotimento a quelle del trauma diretto dovuto all'impatto (scuotimento-impatto). Riteniamo doveroso ricordare che le cadute accidentali nella fascia d'età trattata, in particolare le cadute accidentali dalla propria altezza o le cadute da piccola altezza (letto, divano, braccia del genitore) o alcuni giochi con l'adulto (es. aeroplanino, cavalluccio) non possono in alcun modo determinare, né per dinamica né per intensità di forza necessaria, un quadro clinico così grave come quello osservato nei casi di trauma cranico abusivo, sebbene nella raccolta anamnestica spesso i caregiver, poiché raramente ammettono di aver scosso per senso di colpa o paura, possono inventare eventi accidentali di questo tipo introducendo elementi fuorvianti nella fase di definizione della diagnosi.

\section{Fattori che possono aumentare} lo stress e facilitare reazioni impulsive

Tra i fattori di rischio più spesso indicati dagli autori che possono rappresentare un trigger per l'adulto e predisporre a un trauma cranico abusivo vi è il pianto del bambino, la cui trattazione più diffusa viene rimandata a un paragrafo successivo, ma anche il training all'uso del vasino oppure problematiche mediche come le coliche gassose.

I bambini vittime di scuotimento hanno in genere un'età inferiore all'anno (anche se gli autori estendono ai cinque anni la fascia di età a rischio) e in genere sono più colpiti i soggetti di sesso maschile. Altri fattori di rischio riportati negli studi comprendono una maggiore vulnerabilità del bambino alla nascita (es. basso peso, pretermine, patologia organica congenita) oppure condizioni transitorie successive (es. una malattia infettiva intercorrente oppure una condizione di malessere secondaria alla vaccinazione). Questi elementi possono rendere il bambino più lamentoso, più difficile da comprendere e conseguentemente da trattare, oppure lo rendono fin da subito un bambino diverso dal bambino immaginato o desiderato, meno performante o distante dalle aspettative dell'adulto e quindi meno tollerato e tollerabile nei propri bisogni primari e nella costruzione della relazione, alimentando così un circolo vizioso.

L'altra componente della relazione maltrattante, l'adulto, presenta anch'essa fattori di vulnerabilità, anche se va ricordato che, in modo più evidente rispetto ad altre forme di maltrattamento, le famiglie e i genitori non necessariamente presentano un profilo tipico di fattori di rischio, anzi spesso i genitori vengono descritti come apparentemente adeguati e questa valutazione potrebbe risultare fuorviante per la diagnosi. Questo riscontro si ritrova più frequentemente nel trauma cranico abusivo piuttosto che nel maltrattamento fisico o nel neglect proprio per la natura impulsiva di questa forma di maltrattamento, che insorge in un periodo particolarmente vulnerabile della famiglia e lo rende un "evento possibile" più spesso di quanto non si pensi anche in famiglie senza particolari fattori di rischio. Proprio per questo motivo, come vedremo più avanti, la prevenzione primaria e l'orientamento anticipatorio vanno erogati a tutte le famiglie e non solo alle famiglie cosiddette "vulnerabili o fragili”.

Secondo quanto ricavabile dagli studi, nella maggior parte dei casi l'adulto che scuote è un soggetto di sesso maschile, soprattutto il padre biologico o il patrigno. In casi più rari sono responsabili del maltrattamento la madre naturale, entrambi i genitori contemporaneamente, la baby sitter, o altri parenti a cui è affidato temporaneamente il bambino. Altri fattori di vulnerabilità sono la giovane età del caregiver, la famiglia monoparentale, patologie psichiatriche negli adulti, in particolare la depressione post partum, in misura mino- re l'abuso di sostanze stupefacenti o di farmaci e infine i deficit intellettivi.

Relativamente al contesto, si riportano l'isolamento e/o uno scarso supporto sociale - pensiamo per esempio ai nuclei di migranti con l'impossibilità di usufruire del supporto delle famiglie di origine perché distanti - la presenza di problematiche di coppia, una condizione, anche transitoria, di difficoltà economiche (per esempio la perdita improvvisa del lavoro).

\section{Il pianto del bambino: fisiologia e percezione del caregiver}

Secondo diverse stime pubblicate in letteratura, i bambini, soprattutto dalle 2 settimane fino ai 3-4 mesi circa di età, trascorrono in media dalle 1,5 alle 3 ore al giorno a piangere e tale caratteristica rientra nell'ambito della fisiologia dello sviluppo normale del bambino. Questo dato è un'informazione generalmente poco diffusa nella popolazione e nell'immaginario di molti genitori che si ritrovano d'improvviso a gestire una caratteristica del bambino a cui non erano affatto o poco preparati. Tale comportamento viene interpretato spesso come un malessere del neonato, come se qualcosa non andasse bene: in realtà alla valutazione del pediatra il bambino sta bene e questo può disorientare il genitore. Questo tipo di pianto tende a verificarsi in genere nelle ore serali, e, se persistente, può creare dei sentimenti di frustrazione, impotenza, inadeguatezza, colpa o rabbia nel caregiver, sollecitando possibili reazioni impulsive, soprattutto se associate ad altre condizioni di aumento dello stress come già descritto. La deprivazione prolungata del sonno, la solitudine, l'inesperienza o fattori più gravi come l'uso di sostanze che riducono la tolleranza alla frustrazione. Infine, poiché lo scuotimento provoca l'arresto del pianto, il caregiver tende a reiterare questo comportamento nel tempo.

Un'adeguata informazione sul pianto del lattante e una formazione mirata sulla gestione dello stesso hanno contribuito sensibilmente alla riduzione dell'incidenza dell'AHT nei Paesi in cui si è adottato questo programma di prevenzione primaria e questo obiettivo potrebbe facilmente essere gestito dal pediatra di famiglia nelle prime visite dei neonati.

\section{La pandemia da SARS-CoV-2 e la soglia di vulnerabilità dei nuclei}

Nel corso dell'ultimo anno, a partire da marzo 2020, le misure di isolamento sociale implementate nei Paesi per ridurre la diffusione del Covid-19, hanno imposto alle famiglie l'isolamento forzato al proprio do- 


\section{BOX 1. Pillole di epidemiologia dell'AHT}

Si ritiene che questa sindrome presenti una sottostima dei casi sul piano epidemiologico, poiché i casi clinicamente meno gravi o paucisintomatici possono non giungere mai ai servizi di cura oppure non essere riconosciuti e diagnosticati.

II Centers for Disease Control and Prevention di Atlanta (USA) riporta un dato di incidenza di 30-35 casi ogni 100.000 bambini nati vivi. Negli USA, si stima che 1000-1500 neonati e bambini siano vittime ogni anno di AHT.

In Scozia una registrazione prospettica dei casi di lungo periodo (1996-2008) ha registrato un'incidenza di 33,8/100.000 nati vivi.

L'incidenza del trauma cranico da abuso in Italia è sconosciuta. In Veneto nel corso del 2017, si è registrata un'incidenza di 22 casi ogni 100.000 nati vivi. Da una precedente indagine fatta nella stessa Regione sulle schede di dimissione ospedaliera tra il 2001 e il 2004, si stimava un'incidenza annua di circa 30 casi ogni 100.000 nati vivi.

micilio sia per il lockdown generalizzato sia per misure specifiche di quarantena.

Sebbene l'isolamento e il distanziamento sociale siano risultati misure efficaci nel contenimento della diffusione del virus, hanno portato con sé conseguenze molto significative sul piano sociale, economico e psicologico che hanno contribuito ad aumentare lo stress e conseguentemente la violenza. Tale condizione, infatti, ha acuito le vulnerabilità personali e collettive, limitando contemporaneamente la possibilità di accedere al supporto della famiglia allargata e delle reti informali. Inoltre, la pandemia ha contribuito da un lato ad aumentare lo stress economico, l'instabilità sociale legata alla condizione di emergenza ma anche la riduzione del supporto istituzionale da parte delle diverse agenzie che si occupano di salute e di prevenzione, oltre che in modo specifico di bambini e famiglie sia sul piano sociale che sanitario. In questo contesto si è assistito in tutto il mondo a un notevole aumento della violenza intrafamiliare in tutte le sue forme, sia tra partner sia nei confronti dei bambini. Per esempio, nella provincia di Wuhan, le segnalazioni per violenza domestica sono aumentate di oltre 3 volte nel febbraio 2020 rispetto all'anno precedente. Anche in Europa, non appena si è imposta la quarantena con l'intento di rallentare l'ondata epidemica, il governo italiano ha iniziato a commissariare alcuni alberghi per dare ospitalità a un numero crescente di persone che fuggivano da condizioni di violenza, in modo analogo ad altre nazioni in Europa.

Un recente studio descrittivo inglese ha segnalato un aumento del $1493 \%$ dei casi di AHT nell'area monitorata in un singolo mese: sono stati infatti rilevati ben 10 casi dal 23 marzo al 23 aprile, periodo del lockdown in UK, rispetto a un dato medio precedente di 0,67 casi mensili nei 3 anni precedenti.

Molti autori peraltro ritengono che tale dato sia sottostimato, vista la riduzione generale di accesso ai servizi ospedalieri registrata nel periodo di massima emergenza.
Cosa accade quando si scuote:

fenomeni meccanici e metabolici e patogenesi del danno

Il neonato e il lattante presentano caratteristiche strutturali (anatomiche e fisiologiche) che lo rendono più vulnerabile ai danni da scuotimento, in particolare la letteratura segnala una sproporzione tra le dimensioni del capo e il resto del corpo; un mancato controllo del capo (acquisito completamente a partire dal quarto mese), la mancata o parziale saldatura delle suture craniche; un'incompleta mielinizzazione del parenchima cerebrale che lo rende meno protetto alle forze meccaniche; un'immaturità del processo di autoregolazione della circolazione cerebrale, in caso di alterazioni transitorie sia metaboliche che perfusorie, un'immaturità della barriera ematoencefalica che può essere coinvolta nell'iperpermeabilità dei vasi cerebrali e quindi nel processo di infarcimento emorragico.

Cosa accade quanto un piccolo è sottoposto alle forze di scuotimento? L'effetto immediato, che genera i primi danni macro-e microscopici, è da attribuire alle forze meccaniche esercitate: le brusche accelerazioni e decelerazioni a cui è sottoposto il bambino, in primis il capo e il collo, ma in generale tutto il corpo, provocano, tra gli altri, la rottura dei vasi cerebrali, lo stiramento e la rottura degli assoni, lo stiramento dei vasi retinici così come stirano le metafisi delle ossa lunghe a livello scheletrico.

Una tra le lesioni più comuni è la comparsa di emorragie intracraniche, più frequentemente in sede subdurale (ESD), causate dalla rottura, per trazione, delle vene a ponte e quindi dall'accumulo di sangue tra la dura madre e l'aracnoide (arrivando persino a vere e proprie lesioni da strappamento del vaso che formano un piccolo coagulo, detto lollipopsign). ESD si possono riscontrare in entrambi gli emisferi, nello spazio sopra e sottotentoriale nonché nello spazio subdurale pericerebellare. Inoltre, molto frequentemente, si osservano emorragie nello spazio sottodurale anche a livello spinale (cervicale, dorsale e/o lomba- re), legati in questo caso a movimenti di flesso-estensione repentina del dorso con escursione violenta del cingolo inferiore. Nei casi di reiterazione dell'azione dello scuotere, è possibile documentare ESD sia acuti che cronici, con diversi stadi di degradazione dell'emoglobina (emoglobina, metaemogliobina fino all'emosiderina), oltre che di una riorganizzazione vera e propria dell'ematoma subdurale in strutture più complesse. Dopo la fuoriuscita del sangue dai vasi in fase acuta e l'accumulo dello stesso nello spazio subdurale, altrimenti virtuale, in taluni casi le emazie, le reti di fibrina e i sali di ferro stimolano il reclutamento di macrofagi e plasmacellule che causano la liberazione di citochine proinfiammatorie, le quali a loro volta spingono le cellule gliali a maturare in fibroblasti che, ancorati alle reti di fibrina, si differenziano in cellule epiteliali originanti il tessuto fibrotico cicatriziale e in cellule endoteliali costituenti pareti di neovasi. Questo processo origina le neomembrane, costituite da un tessuto connettivale fibrotico contenente fibroblasti, macrofagi e numerosi capillari sottili, la cui presenza sembra possa aumentare il rischio di risanguinamenti.

Il sangue fuoriuscito nello spazio subdurale contribuisce anche alla genesi di un'alterazione della circolazione del liquor, il cui riassorbimento è alterato e rallentato a livello dei villi aracnoidei per deposito della parte corpuscolata del sangue stravasato. Questa alterazione è tra le principali responsabili, insieme all'aumento di volume dovuto alla fuoriuscita di sangue, dell'aumento abnorme della circonferenza cranica.

Meno frequentemente si osservano emorragie subaracnoidee (ESA), in genere nei casi clinicamente più gravi, che possono avere diversa estensione e che spesso si associano a ESD.

Più di rado si osservano emorragie parenchimali, che sono in genere piccole e associate a traumi da scivolamento. Sono descritte petecchie o piccole lesioni.

Quando allo scuotimento si associa l'impatto, si possono documentare danni parenchimali come contusioni e/o lacerazioni cerebrali che possono verificarsi in sedi sia superficiali che profonde, singole o multiple. Qualora si determini una frattura cranica è possibile la formazione di un'emorragia extradurale (EED).

Le emorragie retiniche (ER) si osservano nell'AHT tra il 40 e l'85\% dei casi, dal polo posteriore fino all'estrema periferia. Esse possono essere preretiniche o subialoidee, situate davanti alla retina; intraretiniche, tra gli strati della retina; sub-retiniche, situate al di sotto della retina. Più di rado, e nei casi più severi, si osservano 
emorragie vitreali. In genere per aspetto, distribuzione, forma e localizzazione sono abbastanza tipiche e si distinguono da quelle osservate in altre condizioni patologiche.

Le emorragie subretiniche e intraretiniche tipicamente possono essere puntiformi (dot), a macchia (blot) o a fiamma (flame). Le emorragie sub- e intraretiniche tendono a guarire nel giro di alcune settimane, mentre le emorragie più gravi (preretiniche o vitreali) necessitano di diverse settimane o mesi per riassorbirsi, impedendo alla luce di filtrare e creando di fatto una deafferentazione visiva.

Le ER nei casi di AHT possono essere poche emorragie puntiformi o a macchia al polo posteriore o anche all'estrema periferia, oppure il quadro si può presentare con emorragie estese e confluenti che ricoprono l'intera superficie della retina e risparmiano solo l'ora serrata. Nei casi più severi sono stati descritti anche retinoschisi traumatiche o pieghe retiniche perimaculari.

Sebbene più frequentemente i danni a livello della retina siano bilaterali e generalmente simmetrici, non è infrequente riscontrare lesioni asimmetriche e anche monolaterali.

Anche a livello retinico, data la possibile ripetitività degli scuotimenti osservata nella maggior parte dei casi, all'esame oftalmoscopico è possibile registrare la presenza contemporanea di emorragie retiniche pregresse (già quasi assorbite con il centro più chiaro) e recenti.

Il meccanismo di formazione delle ER è di tipo meccanico e lo stravaso (dal basso verso l'alto) avviene per trazione-detrazione del bulbo oculare vs il fascio vascolonervoso.

Il danno di origine meccanica, tuttavia, non è l'unico responsabile di tutto il complesso quadro lesivo che si riscontra nei bambini con diagnosi di AHT: il quadro encefalopatico di solito descritto in questi pazienti è prodotto per alterazioni metaboliche di tipo ipossico-ischemico derivanti dall'alterazione della funzione dei centri del respiro e del battito, provocata a sua volta dallo stiramento del bulbo e dall'alterazione dei meccanismi di regolazione della circolazione cerebrale. La trazione del bulbo del tronco encefalico comporta infatti bradicardia, che può arrivare fino all'arresto cardiaco, e bradipnea, fino all'apnea. Bradipnea e bradicardia determinano a loro volta una riduzione dell'afflusso di sangue al cervello e una scarsa ossigenazione dello stesso, provocando edema citotossico intracellulare, con ipossia, ipercapnia, acidosi e alterazione dell'autoregolazione vascolare cerebrale, a cui si associa un edema extracellulare vasogenico. Il quadro di edema contribuisce ad aumentare la pressione intracranica e quindi il flusso ematico cerebrale cala danneggiano il microcircolo con conseguente ischemia neuronale, responsabile di nuova ipossia, con un meccanismo che si automantiene.

Al neuroimaging è possibile documentare delle vere e proprie aree ischemiche, sia focali che di importanti porzioni dell'encefalo, oltre che la presenza di danno assonale diffuso.

Il principale esito a cui portano tutti i meccanismi patogenetici sin qui descritti, in modo particolare i danni ipossicoischemici, è l'atrofia cerebrale, insorgente dopo 15-30 giorni dallo scuotimento, che si riconosce indirettamente per un aumento del volume dei ventricoli cerebrali oltre che per un aspetto ulegirico delle circonvoluzioni cerebrali caratterizzato da assottigliamento della corteccia, forma a fungo delle circonvoluzioni e con maggiore distanziamento dalle altre.

Il quadro è aggravato dal fatto che l'AHT è un maltrattamento che tende a riprodursi nel tempo, perciò alla diagnosi i bambini che hanno subito più scuotimenti presenteranno quadri emorragici complessi all'esame del fundus oculi e al neuroimaging cerebrale, associati a una concomitante atrofia cerebrale ormai avanzata.

Infine, sebbene di gran lunga meno gravi in termini di outcome, ricordiamo che le fratture sono un segno clinico frequente nelle vittime di AHT, documentate dal 25 al 40\% dei casi e, ove presenti, di grande supporto per la definizione diagnostica. Oltre alle fratture craniche nello scuotimento-impatto, si osservano frequentemente fratture delle diafisi e delle metafisi delle ossa lunghe e fratture costali da compressione del torace (ricordiamo che la rianimazione cardiopolmonare non è quasi mai responsabile di fratture costali).

\section{Associazione con altre forme di maltrattamento}

L'AHT, come più volte sottolineato, è una forma di maltrattamento che presenta caratteristiche peculiari e che insorge in fasi specifiche della vita del bambino. Tale sottoforma si associa non così di rado ad altre forme di maltrattamento che presentano fattori di rischio e meccanismi "patogenetici” simili in termine di relazione maltrattante. Le associazioni più frequentemente descritte si osservano con il maltrattamento fisico, per esempio con il riscontro di fratture provocate con altre forze come la torsione o la piegatura oppure la presenza di ustioni, e con il neglect precoce, altra forma di maltrattamento che esprime la "difficoltà" del caregiver nella gestione di un nuovo nato.

Infine, un'altra forma di maltrattamento emergente spesso associata all'AHT è il chemical abuse (es. l'esposizione a farmaci o tossici) sia per contaminazione ambientale che per somministrazione volontaria. Questa forma di maltrattamento si osserva più spesso nei nuclei in cui esiste in maniera nota o sconosciuta un disturbo di dipendenza da sostanze. L'utilizzo della sostanza da parte dell'adulto può da un lato facilitare comportamenti impulsivi dello stesso (pensiamo per esempio alla cocaina che riduce la tolleranza allo stress e facilita il passaggio all'atto) e dall'altro favorire l'utilizzo di farmaci o sostanze illecite per modulare il comportamento del bambino (per esempio utilizzare ansiolitici, eroina o metadone per favorire il sonno del bambino).

Per questi motivi, nel caso di riscontro di ecchimosi o di una frattura in un piccolino nei primi mesi di vita, oppure in caso di esposizione a sostanze, bisogna sempre effettuare un work up diagnostico per escludere la presenza di AHT (anche se clinicamente non immediatamente manifesto).

\section{Caratteristiche cliniche dei casi:}

segni e sintomi di sospetto

L'AHT può presentarsi con sintomi e segni molto diversi rendendo insidioso il percorso diagnostico: può esordire come una Brief Resolved Unexplained Event (BRUE), oppure accedere a un servizio di cure primarie o dal pediatra di famiglia per una macrocrania in apparente completo benessere, può presentare ridotta interazione, mancanza di sorriso sociale, scarso feeding oppure scarsa crescita.

Quando la sintomatologia è grave o gravissima, il bambino si presenta con pallore, ipotonia, cianosi, mancata responsività agli stimoli, bradicardia, bradipnea, oppure come un episodio critico similepilettico (può presentare una fontanella anteriore tesa e pulsante), in altri il bambino appare in una condizione tale da far sospettare una condizione grave, ma non apparentemente a rischio di vita (mostrando pianto inconsolabile, irritabilità, vomiti ripetuti, agitazione), in altri ancora il bambino giunge a valutazione in condizioni generali discrete o addirittura buone, per crescita anomala della circonferenza cranica, scarso feeding o anemia. Infine, è possibile anche osservare gli esiti di pregressi scuotimenti mai diagnosticati (ritardo dello sviluppo, deficit motori o visivi). Nella maggior parte dei casi i segni clinici sono così aspecifici che il curante può scambiarli come segni di una banale virosi o un'altra patologia minore. 


\section{BOX 2. L'iter diagnostico}

1. Obiettività generale. Lesioni cutanee anche minime (es. segni di presa) 0 altre lesioni più gravi (es. ustioni).

2. Parametri auxologici. Particolare attenzione alla circonferenza cranica (considerando l'andamento dalla nascita), molto spesso aumentata in maniera sproporzionata e disarmonica rispetto a peso e altezza.

3. Indagini bioumorali. Primo livello: profilo ematologico, la formula leucocitaria, valutazione della coagulazione, stato nutrizionale, indici di flogosi. Secondo livello: screening emo-coagulativo completo, studio del metabolismo del rame, acidi organici urinari e aminoacidi plasmatici, ed altri eventuali accertamenti specifici volti a escludere altre diagnosi differenziali (vedi infra). Marker sierici di trauma cranico (NSE e proteina SB100), positivi fino a 48-72 ore dal trauma.

4. Neuroimaging. RMN cerebrale e spinale (comprensiva dello studio dei vasi mediante AngioRMN) come gold standard da eseguire immediatamente e a distanza di 7 e 14 giorni per monitorare I'evoluzione del quadro. Se disponibilie, MRS (Magnetic Resonance Spectroscopy), DWI, fasterRMN (per le anomalie parenchimali), DTI (per lo studio trattografico dei fasci di sostanza bianca, per valutare precocemente il danno e la prognosi).

5. Esame oftalmoscopico accurato del fondo dell'occhio, con esplorazione anche della estrema periferia della retina, e imaging digitale della retina (Ret Cam).

6. Esame radiografico dello scheletro per segmenti e un esame scintigrafico.

7. Esame tossicologico su capello.

8. Anamnesi. Raccogliere con scrupolo le notizie relative alle $48 \mathrm{~h}$ precedenti l'episodio. E poi informazioni riguardo alla nascita, alle principali tappe di sviluppo (nell'a. patologica remota e prossima particolare attenzione a sintomi quali vomito senza febbre e/o diarrea, irritabilità, scarsa alimentazione, crescita anomala del capo, pianto insistente, inappetenza, sonni prolungati). Nell'a. familiare attenzione a struttura e composizione della famiglia, sul lavoro e i legami che la famiglia ha nel proprio interno, eventi stressanti più o meno recenti come nuove nascite, lutti, perdite di lavoro, traslochi, presenza di altri figli minori con scarsa spaziatura, ecc.

Quando l'episodio di scuotimento non porta a un quadro clinico di BRUE, anche per la mancata saldatura delle ossa del cranio, superata la fase acuta e subacuta, si osserva una stabilizzazione del quadro, con un aumento della circonferenza cranica esitante in macrocrania come unico segno manifesto.

Quadri clinici severi, vista la gravità e la conseguente probabilità di impiego di tecniche di neuroimaging, saranno più facilmente diagnosticati rispetto a quadri clinici lievi, comunemente sottoposti alla sola osservazione prolungata, che saranno diagnosticati solo grazie all'esperienza del professionista.

Anche il riscontro di altre forme di maltrattamento in questo periodo di vita deve far sospettare la concomitante diagnosi di AHT, per esempio un lattante con frattura, con condizione di neglect oppure con esposizione a stupefacenti o chimici.

\section{Trattamento e management}

La maggior parte del trattamento nei casi di AHT è di supporto, con monitoraggio delle funzioni vitali (può essere necessaria l'intubazione e la ventilazione meccanica), monitoraggio della pressione intracranica, eventuale trattamento chirurgico.

Alcuni bambini affetti da AHT necessitano di intervento neurochirurgico in urgenza per garantire la sopravvivenza o migliorare l'outcome, per esempio con craniotomie decompressive in caso di erniazione, deterioramento neurologico o in paziente non responsivi a terapia medica nelle cure intensive.
Nella maggior parte dei casi, tuttavia, il danno intracranico si presenta in forma subacuta o cronica e non richiede un intervento in urgenza. Rispetto agli interventi elettivi per prevenire le complicanze (es. riorganizzazione degli ematomi e formazioni di neomembrane) in letteratura sono descritti diversi approcci: da interventi minimamente invasivi con un drenaggio della raccolta (burr hole o twist-drill holes) fino a interventi di craniotomia con membranectomia e cranioplastica. Non esiste attualmente un approccio condiviso a livello internazionale in quanto le diverse tecniche hanno dimostrato di avere sia vantaggi che svantaggi. Nella nostra esperienza, l'approccio elettivo in fase subacuta con membranectomia e cranioplastica ha mostrato ottimi risultati in termini di outcome complessivo a distanza (rivalutazione a 5 anni), con un quoziente di sviluppo migliore nei bambini sottoposti a intervento rispetto a quelli trattati con altri tipi di approcci.

\section{Prognosi del bambino con SBS}

Un terzo dei bambini con AHT avrà conseguenze minime a lungo termine, un terzo presenterà disabilità cognitive e motorie severe e il rimanente dei casi morirà a causa delle lesioni; in genere la gravità delle sequele è correlata all'estensione delle lesioni cerebrali. Le caratteristiche che in fase acuta influenzano maggiormente l'outcome sono un basso punteggio alla scala di Glasgow oppure la presenza di episodi critici; inoltre, risultano rilevan- ti anomalie del parenchima cerebrale a 3 mesi dalla diagnosi, la precocità degli scuotimenti nei primi mesi di vita oltre che la presenza di danni di tipo ischemico e un quadro retinico particolarmente compromesso.

\section{Sviluppo neurocognitivo}

L'outcome neurologico riflette la tipologia e l'estensione dei danni cerebrali risultante dall'insulto ipossico. Alcuni bambini presentano gravi paralisi cerebrali con tetraplegie ed emiplegie, epilessia, altri presentano quadri neurologici più sfumati. Le lesioni causate da un AHT, comprensivo della sottoforma SBS, comportano compromissioni dello sviluppo psicomotorio, cognitivo e comportamentale fino alla disabilità. Tanto più il danno iniziale è rilevante e tanto più precocemente avviene (entro il primo anno di età) quanto peggiore sarà l'esito dello sviluppo. Infatti, bambini di età inferiore a un anno al momento del trauma hanno più probabilità di sviluppare disturbi del neurosviluppo a lungo termine, rispetto ai bambini di due anni, con un'incidenza che accresce all'aumentare dell'età, soprattutto in età scolare, periodo evolutivo in cui le richieste di apprendimento e sociali sono maggiori. In particolare, a un generale ritardo dello sviluppo, da un punto di vista neurologico si riscontra un quadro di epilessia, che emerge già dopo 3 mesi dal trauma, prima di altre manifestazioni. I deficit neurologici compromettono lo sviluppo psicomotorio che si può manifestare con ipotonia, emiplegia spastica, quadriplegia, atassia, distonia. Le performance intellettive di questa popolazione di bambini sono significativamente inferiori rispetto ai controlli. Da un punto di vista cognitivo le funzioni maggiormente compromesse includono ritardi del linguaggio (in maniera particolare nelle prove di fluenza che comprendono parole simili dal punto di vista fonetico e non semantico, nella comprensione di istruzioni in cui è necessario ritenere ed elaborare intere istruzioni verbali al fine di eseguire il contenuto di quell'istruzione, nel linguaggio espressivo e recettivo, e nelle prove di vocabolario), deficit di memoria (memoria visiva e verbale, working memory, memoria retrospettiva); una particolare attenzione va posta al deficit di memoria di lavoro poiché dimostra probabili lentezze nei processi di codifica e ritrovamento dell'informazione. Questa osservazione suggerisce che i bambini che hanno subito un trauma abbiano maggiore difficoltà nell'organizzare e classificare con efficienza gli elementi di informazione presentati, prima di immagazzinarli nella loro memoria a lungo termine. Questo 
BOX 3. Le principali diagnosi differenziali

\section{- Trauma post partum.}

Traumi accidentali.

- Patologie infettive (es. malaria, meningiti virali, encefalite erpetica, otiti medie croniche, sinusiti croniche, toxoplasmosi congenita, rottura di aneurisma micotico).

- Neoplasie (es. leucemia, linfoma della dura madre, sarcoma subdurale, meningioma, metastasi meningee, tumori solidi al SNC, carcinoma e papilloma dei plessi corioidei, xantogranuloma gigante).

- Malformazioni congenite (es. MAV intracraniche, aneurisma cerebrale, cisti aracnoidee, sindrome di Osler-Weber-Rendu, idrocefalo congenito, encefalocele e meningocele).

- Disordini ematologici e dell'assetto coagulativo (es. emofilia A e B, deficienza del fattore V, deficienza del fattore XII, malattia di Von Willebrand, deficit di vitamina K, disfibrinogenemia congenita, porpora trombocitopenia idiopatica, CID).

- Disordini metabolici genetici (es. anemia falciforme, osteogenesi imperfetta, sindrome di Ehlers-Danlos, sindrome di Marfan, malattia di Menkes, sindrome di Alagille, omocistinuria, aciduria glutarica di tipo I, iperostosi interna del frontale).

- Deficit vitaminici gravi.

- Vasculiti e patologie autoimmuni (LES, malattia di Kawasaki, malattia di Moyamoya, granulomatosi di Wegener, malattia di Behçet).

processo conta sul funzionamento corretto della memoria di lavoro ma anche sull'organizzazione mentale.

Possono esservi inoltre deficit di attenzione, in maniera particolare nell'attenzione uditiva e attenzione divisa (quel processo necessario per mantenere e gestire istruzioni multiple, direttamente correlato all'efficienza dell'esecutivo centrale), deficit visuomotori (integrazione visuomotoria, abilità fine e grosso motoria, compromissioni grafiche) e disturbi dell'apprendimento.

Appaiono pertanto deficitarie le funzioni che comprendono memoria di lavoro, attenzione condivisa, ragionamento, organizzazione e pianificazione mentale, alternanza e inibizione mentale, particolarmente nella espressione verbale. Alcuni ricercatori suggeriscono che queste abilità dovrebbero essere raggruppate sotto il termine "funzioni esecutive", funzioni che sono direttamente collegate all'integrità dei lobi frontali. Dal punto di vista comportamentale le problematiche maggiormente riscontrate sono le difficoltà dell' $i$ nibizione comportamentale, difficoltà a inibire il pensiero che causa una mancata risoluzione del problema, stati di agitazione, mancanza di tolleranza alla frustrazione, mancanza di autocontrollo e incapacità ad adattarsi al cambiamento, aggressività, impulsività, irritabilità, comportamenti stereotipati, fino a comportamenti autolesivi e di autostimolazione. Il principale correlato neurale è la disfunzione del lobo frontale, in associazione e fattori ambientali. In un recente studio in cui i bambini sono stati seguiti fino all'età di 11 anni, si è riscontrato un incremento nella presenza delle problematiche comportamentali ri- $^{-}$ spetto a quanto rilevato a 5 anni. In un altro studio a un follow up fino ai 3 anni, si è osservato che i bambini che presentavano una gravità moderata del trauma soffrivano maggiormente di problemi internalizzanti a confronto con i bambini che al momento del trauma presentavano un danno di lieve entità.

I deficit precedentemente descritti, in particolare quelli visuomotori e linguistici, presentano ricadute anche a livello delle abilità sociali, comportando una minor integrazione e minore capacità adattive, in termini di comunicazione, autonomia quotidiana e socializzazione. È stato riscontrato che questi bambini a seguito delle problematicità possano necessitare di servizi educativi speciali durante il percorso scolastico. È difficile definire con certezza se tali conseguenze a livello cognitivo e comportamentale siano dovute ai danni neurologici in sé o alla storia di maltrattamento vissuta da questi bambini: ai deficit, alla qualità del parenting, l'am- biente emotivo e le relazioni affettive presenti prima e dopo il maltrattamento influenzano infatti le conseguenze a lungo termine.

\section{Vista}

Nei bambini con AHT si documentano deficit visivi più o meno marcati (riduzione dell'acuità visiva, riduzione del campo visivo, alterazioni della motricità oculare, strabismo) e nei casi più gravi il cortical visual impairment (per danno diretto alla corteccia visiva o per deafferentazione sensoriale nella finestra critica di sviluppo causata dalla presenza di vaste emorragie retiniche).

\section{Prevenzione}

Secondo numerosi studi, il trauma cranico da abuso e lo scuotimento sono tra le forme di maltrattamento che hanno mostrato di beneficiare notevolmente di campagne in termini di riduzione dell'incidenza. Esistono numerosi esempi (per esempio in USA, Australia, Giappone, UK) con progetti sia di informazione sui danni da scuotimento sia di informazione sul pianto di neonato e lattante (per esempio il PURPLE crying) oltre che sulle modalità di gestione del pianto, con vere e proprie strategie comportamentali su come calmare il piccolo o come fronteggiare la frustrazione e la rabbia scatenate da un pianto inconsolabile.

Anche in Italia, nel corso dell'anno 2017, ha preso avvio una campagna informativa di prevenzione primaria sullo scuotimento (nonscuoterlo.it), promossa dalla ONG Terre des Hommes.

Esistono inoltre numerosi altri esempi di campagne e strategie di prevenzione, quali le visite domiciliari prima e dopo la nascita per favorire la genitorialità (programmi di home visiting), anch'esse dimostratesi molto efficaci nella promozione di una buona relazione genitori-bambino e in grado di prevenire altre forme gravi di maltrattamento come il neglect.

melissa.rosarizzotto@aopd.veneto.it

La bibliografia è consultabile online. 


\section{Bigliografia}

1. Babl FE, Pfeiffer $H$, Kelly $P$, et al. Paediatric Research in Emergency Departments International Collaborative (PREDICT). Paediatric abusive head trauma in the emergency department: A multicentre prospective cohort study. J Paediatr Child Health. 2020;56(4):615-621.

2. Choudhary AK. Understanding the importance of spinal injury in abusive head trauma (AHT). Pediatr Radiol. 2020;50(1):15-16.

3. American Academy of Pediatrics. Consensus Statement: Abusive Head Trauma in Infants and Young Children. Pediatrics. 2018;142(2):e20181504.

4. Duhaime AC, Christian CW. Abusive head trauma: evidence, obfuscation, and informed management. J Neurosurg Pediatr. 2019;24(5):481-488.

5. Eismann EA, Theuerling J, Cassedy A, et al. Early developmental, behavioral, and quality of life outcomes following abusive head trauma in infants. Child Abuse \& Neglect 2020;108;104643.

6. Hymel KP, Wang M, Chinchilli VM, et al. Estimating the probability of abusive head trauma after abuse evaluation. Child Abuse Negl. 2019;88:266-274.

7. Iqbal O'Meara AM, Sequeira J, Miller Ferguson N. Advances and Future Directions of Diagnosis and Management of Pediatric Abusive Head Trauma: A Review of the Literature. Front Neurol. 2020;11:118.

8. Jackson JE, Beres AL, Theodorou CM, et al. Long-term Impact of Abusive Head Trauma in Young Children: Outcomes at 5 and 11 Years Old. J Pediatr Surg. 2021 Feb 19:S0022-3468(21)00153-6

9. Sidpra J, Abomeli D, Hameed B, et al. Rise in the incidence of abusive head trauma during the COVID19 pandemic. Arch Dis Child. 2021;106(3):e14.

10. Janson S. Towards a deeper understanding of abusive head trauma. Acta Paediatr. 2020;109(7):12901291.

11. Kelly P. The economic cost of pediatric abusive head trauma. Child Abuse Negl. 2019;93:13-14.

12. Kennedy JM, Ma J, Lyden ER, et al. Abusive Head Trauma and a Delay in Presentation for Care. Pediatr Emerg Care. 2020; Epub ahead of print, doi: 10.1097/PEC.0000000000002197.

13. Lind $K$, Toure $H$, Brugel $D$, et al. Extended follow-up of neurological, cognitive, behavioral and academic outcomes after severe abusive head trauma. Child Abuse Negl. 2016;51:358-67.

14. Lindberg DM, Dubowitz H, Alexander RC, et al. The "New Science" of Abusive Head Trauma. Int J Child Maltreat. 2019;2(1-2):1-16.

15. Narang SK, Fingarson A, Lukefahr J, et al. Abusive Head Trauma in Infants and Children. Pediatrics. 2020;145(4):e20200203. 
16. Nuño $M$, Ugiliweneza $B$, Zepeda $V$, et al. Long-term impact of abusive head trauma in young children. Child abuse \& neglect. 2018;85:39-46.

17. Porto L, Bartels MB, Zwaschka J, et al. Abusive head trauma: experience improves diagnosis. Neuroradiology. $2021 ; 63(3): 417-430$

18. Rabbitt AL, Kelly TG, Yan K, et al. Characteristics associated with spine injury on magnetic resonance imaging in children evaluated for abusive head trauma. Pediatr Radiol. 2020;50(1):83-97.

19. Rebbe R, Mienko JA, Martinson ML. Incidence and Risk Factors for Abusive Head Trauma: A PopulationBased Study. Child Abuse Rev. 2020;29(3):195-207.

20. Simonnet H, Laurent-Vannier A, Yuan W, et al. Parents' behavior in response to infant crying: abusive head trauma education. Child Abuse Negl. 2014;38(12):1914-22.

21. Storz MA. Child abuse: a hidden crisis during COVID- 19 quarantine. J Paediatr Child Health. 2020;56(6):990-991.

22. Weldy E, Shimoda A, Patnaik J, et al. Long-term visual outcomes following abusive head trauma with retinal hemorrhage. J AAPOS. 2019;23(6):329.e1-329.e4. 